\title{
Double Trouble: Heterotopic Pregnancy
}

\author{
${ }^{1}$ Shruthi Krishnamoorthy, ${ }^{2} \mathrm{G}$ Usha Rani, ${ }^{3} \mathrm{O}$ Syamala, ${ }^{4}$ Rukshana, ${ }^{5}$ Naveen Alexander
}

\section{ABSTRACT}

A heterotopic pregnancy is defined as the presence of a combined intrauterine and ectopic pregnancy. Its estimated incidence is as between $1 / 7000$ and $1 / 30,000$ pregnancies. It is also reported to be as high as $1 \%$ after the use of assisted reproductive technology. Heterotopic pregnancies are diagnostic and therapeutic challenges for obstetricians. If they continue without diagnosis, a life-threatening situation may occur even when timely surgical intervention with laparotomy is performed. Here, we report a case series of three patients having three different scenarios, who were diagnosed with heterotopic pregnancies in the first trimester and managed successfully.

Keywords: Heterotopic pregnancy, Ectopic pregnancy, Intrauterine pregnancy, Laparotomy, Rupture.

How to cite this article: Krishnamoorthy S, Rani GU, Syamala O, Rukshana, Alexander N. Double Trouble: Heterotopic Pregnancy J South Asian Feder Obst Gynae 2014;6(3):167-170.

\section{Source of support: Nil}

\section{Conflict of interest: None}

\section{INTRODUCTION}

A coexistence of an extra- and intrauterine pregnancy is defined as a heterotopic pregnancy. ${ }^{2,4,6}$ With increasing use of assisted reproductive techniques, the incidence of heterotopic pregnancy is also shown to increase. Aside from the difficulty of diagnosing ectopic pregnancy, management can be difficult and may be life threatening, even when surgical intervention with laparotomy is performed. ${ }^{2}$ Heterotopic pregnancy must be considered in a patient who has a confirmed intrauterine pregnancy but presents with abdominal pain and bleeding per vaginum. This study describes heterotopic pregnancy in three patients, two of them who conceived with the aid

\footnotetext{
${ }^{1}$ Postgraduate, ${ }^{2}$ Professor, ${ }^{3,5}$ Associate Professor

${ }^{4}$ Assistant Professor

${ }^{1-4}$ Department of Obstetrics and Gynecology, Sri Ramachandra Medical University, Chennai, Tamil Nadu, India

${ }^{5}$ Department of General Surgery, Sri Ramachandra Medical University, Chennai, Tamil Nadu, India
}

Corresponding Author: Shruthi Krishnamoorthy, Postgraduate, Department of Obstetrics and Gynecology, Sri Ramachandra Medical University, Chennai, Tamil Nadu, India Phone: 091-9962589993, e-mail: krishruthi@gmail.com of ovulation induction and one spontaneous conception and their course of events.

\section{CASE SERIES}

\section{Case 1}

A 23-year-old woman, primigravida, at 6 weeks and 6 days of gestational age, conceived after 3 cycles of ovulation induction with clomiphene citrate, was referred to the casualty, with complaints of pain in left iliac fossa. Patient had one episode of spotting per vaginum 10 days prior. Ultrasound revealed, heterotopic pregnancy of a single live intrauterine gestation of 6 weeks and 6 days, with a left adnexal ectopic pregnancy, with minimal free fluid in the pouch of Douglas. Routine investigations, hemoglobin was 11.8 , beta subunit of human chorionic gonadotropin was 86,536 . Two units of O positive packed cells were reserved. Pulse-82/min, blood pressure-110/60, $\mathrm{SpO}_{2}-99 \%$ on room air. Per abdomen was soft, no tenderness. No bleeding per vaginum, per speculum, Os closed. Emergency laparoscopy was planned as she was hemodynamically stable. After carefully explaining to the patient and attenders about the risk of abortion, possibility of harm to the fetus in utero due to anesthetic effects, laparoscopy was proceeded. About $600 \mathrm{ml}$ of hemoperitoneum was present, and the same removed using suction. Findings suggestive of left ampullary tubal rupture. Hence, left salphingectomy was proceeded, using bipolar cautery. Right tube appeared normal and both ovaries appeared normal. One unit packed cell transfused intraoperatively. Post procedure vitals were stable, and she continued the intrauterine pregnancy. She came for regular antenatal check-ups. Rest of the antenatal period was uneventful.

At 38 weeks and 4 days, on routine antenatal evaluation, she presented with oligohydramnios with amniotic fluid index of $5.7 \mathrm{~cm}$. In view of previously managed heterotopic pregnancy and oligohydramnios, due to maternal demand, elective lower segment cesarean section was performed. Patient delivered a healthy baby boy weighing $3.12 \mathrm{~kg}$ with an Apgar of 8/10,9/10. After 1 year and 2 months, she conceived again and underwent an uneventful pregnancy and delivered a baby girl weighing $2.95 \mathrm{~kg}$ and also underwent right tubal sterilization along with elective lower segment cesarean section. 


\section{Case 2}

A 27-year-old woman, primigravida, at 9 weeks of gestational age, conceived by ovulation induction with clomiphene citrate, was referred to the casualty, with complaints of severe lower abdomen pain. Patient had bleeding per vaginum last 5 hours. Dating scan done at 7 weeks revealed, a single live intrauterine gestation only. Ultrasound pelvis was done after 2 weeks, in view of lower abdominal pain, showed right-sided ruptured ectopic and a single live intrauterine gestation corresponding to 8 to 9 weeks with moderate hemoperitoneum. Features suggesting of heterotopic pregnancy. A repeat ultrasound pelvis done at our institution after 3 hours from the previous scan showed right-sided ruptured ectopic with a massive hemoperitoneum along with a single intrauterine gestation with no cardiac activity, confirming heterotopic pregnancy with missed abortion. On examination, temperature $98.6^{\circ} \mathrm{F}$, pulse rate $112 / \mathrm{min}$, blood pressure $90 / 60 \mathrm{~mm} \mathrm{Hg}$, respiratory rate $20 / \mathrm{min}$ pallor ++. No pedal edema, cardiovascular and respiratory systems normal. Per abdomen, diffuse tenderness present in lower abdominal region and abdominal distension present. Per speculum examination, bleeding present through os. Per vaginal examination. Cervix pointing downwards, uterus anteverted, 8 to 10 weeks size, cervical motion tenderness present, right forniceal fullness and tenderness present, left fornix free.

Investigations, revealed a hemoglobin of $6.3 \mathrm{gm} / \mathrm{dl}$, beta subunit of human chorionic gonadotropin of 92,243 , INR of 1.08. Renal function tests, blood urea nitrogen of $8 \mathrm{mg} / \mathrm{dl}$ and serum creatinine of $0.7 \mathrm{mg} / \mathrm{dl}$. Four units of O positive packed cells were reserved. In view of hemodynamic unstability, emergency laparotomy proceeded. Intraoperatively (Figs 1A and B), $900 \mathrm{ml}$ hemoperitoneum was present. Two hundred gram clots removed.

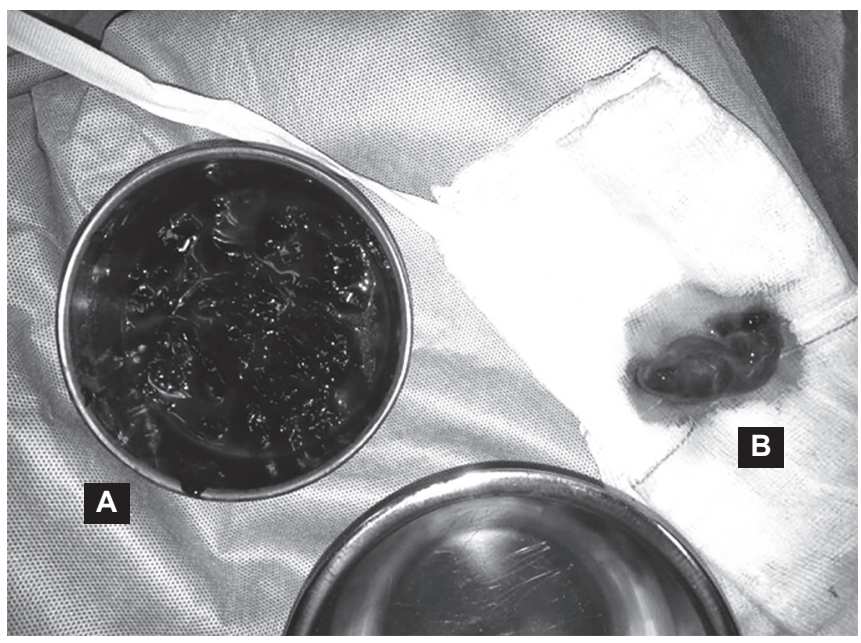

Figs 1A and B: (A) Ruptured right ectopic pregnancy with hemoperitoneum (200 gm clots) and (B) right fallopian tube and products of conception
Right-sided ruptured ectopic of size $3 \times 2 \mathrm{~cm}$ noted in the fimbrial end, for which right salpingectomy was done. Left tube and both ovaries appeared normal. Suction evacuation was performed for missed abortion. Three units of packed cells were transfused intraoperatively. Post procedure, patient had no complications, hence, discharged on postoperative day 5 .

\section{Case 3}

A 29-year-old woman, gravida 2 ectopic 1 , at 7 weeks and 6 days of gestational age, spontaneous conception, presented to the antenatal outpatient department. History of laparoscopic left salpingectomy done in view of left ruptured ectopic pregnancy.

Patient had no complaints of bleeding per vaginum or pain abdomen. On routine scan, heterotopic pregnancy of a single live intrauterine gestation with a live right ectopic pregnancy was diagnosed. Investigations revealed a hemoglobin of $13.3 \mathrm{gm} / \mathrm{dl}$. Beta subunit of human chorionic gonadotropin of 94,933 . Two units of B positive packed cells were reserved. Pulse-78/min, blood pressure $-100 / 70 \mathrm{~mm} \mathrm{Hg}, \mathrm{SpO}_{2}-99 \%$ on room air. Per abdomen was soft, no tenderness. No bleeding per vaginum, per speculum, Os closed. She was admitted in the labor ward for observation. Vitals and abdominal girth closely monitored. After carefully explaining to the patient and attenders about the risk of abortion, possibility of harm to the fetus in utero due to anesthetic effects, laparoscopy was proceeded as she was hemodynamically stable. Intraoperatively (Figs 2A and B), left tube was absent. Right tube had a $6 \times 4 \mathrm{~cm}$ mass with features suggestive of impending rupture. Right and left ovaries were normal. Using bipolar cautery right salpingectomy done. Perfect hemostasis secured (Figs 3A and B). The ectopic pregnancy was removed in toto from laparoscopic $10 \mathrm{~mm}$ port site (Fig. 4).

Post procedure, vitals were stable, and she continued the intrauterine pregnancy. At 11 weeks and 4 days, she presented to the casualty with one episode of spotting per vaginum. She was admitted, subjected to bed rest for 3 days. No further episodes of spotting per vaginum, hence discharged. Now, she is now 20 weeks of gestational age and is continuing the pregnancy without any complications.

\section{DISCUSSION}

Heterotopic pregnancy ${ }^{4,9}$ was first described by Duverney in 1708. The majority of heterotopic pregnancy cases are diagnosed late. Significant morbidity and occasional mortality have been reported as a result of a delay in diagnosis. ${ }^{4}$ As no single investigation can predict the presence 

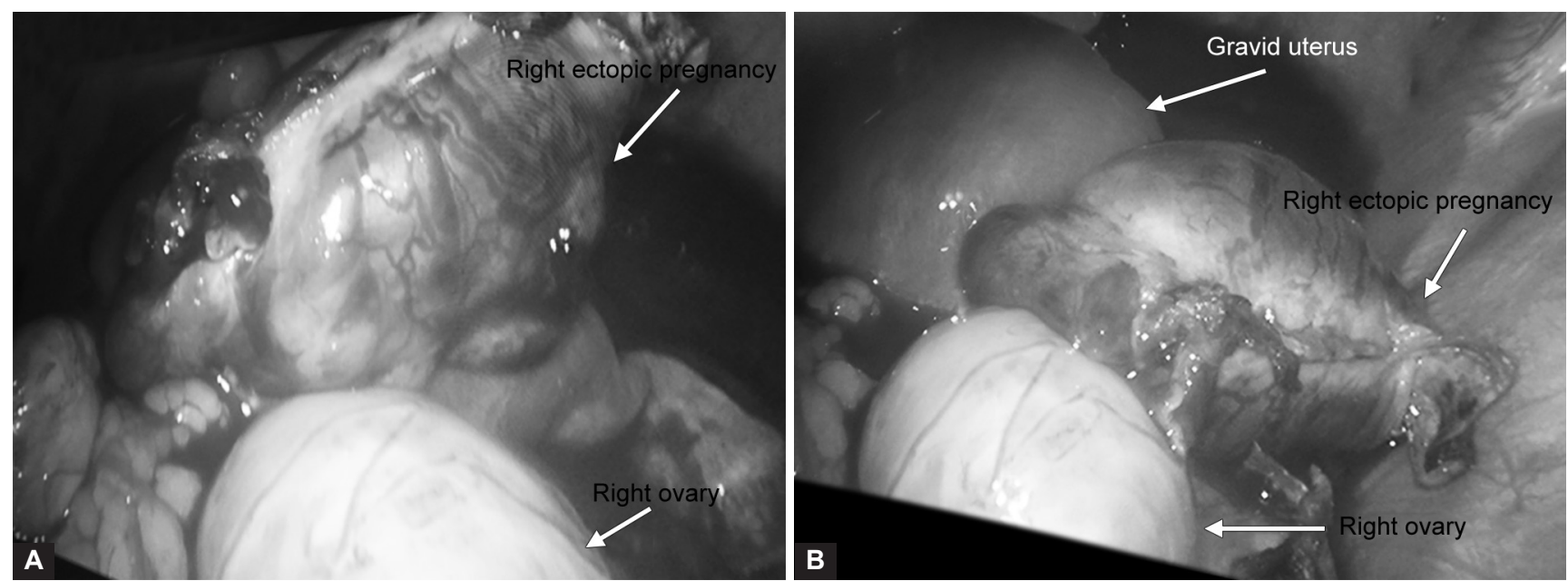

Figs 2A and B: Laparoscopic findings: Right ectopic pregnancy of size $6 \times 4 \mathrm{~cm}$. Uterus corresponding to 8 weeks in size, right normally appearing ovary. Minimal hemoperitoneum (intraoperative photograph at Sri Ramachandra Medical College, Department of Obstetrics and Gynecology)
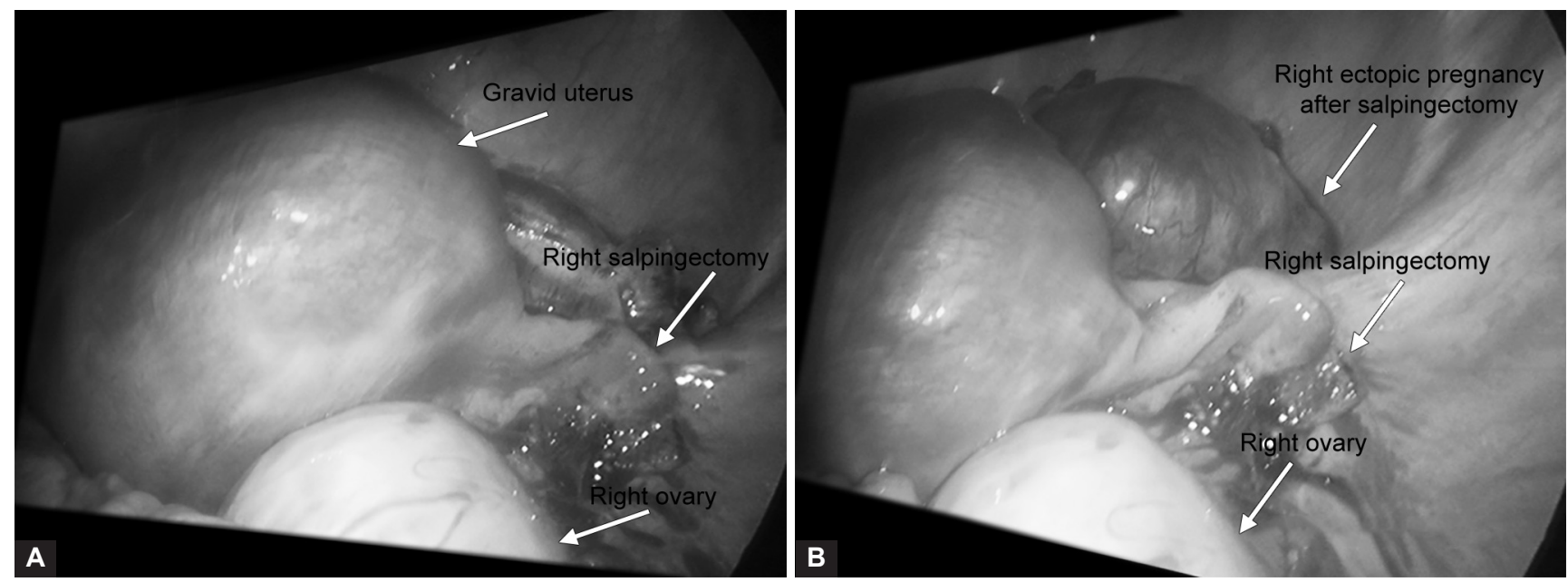

Figs 3 A and B: Heterotropic pregnancy of the right fallopian tube. Removed laparoscopically using bipolar cautery, and placed anteriorly. Perfect hemostasis secured at the cut ends of the fallopian tube (intraoperative photograph at Sri Ramachandra Medical College, Department of Obstetrics and Gynecology)

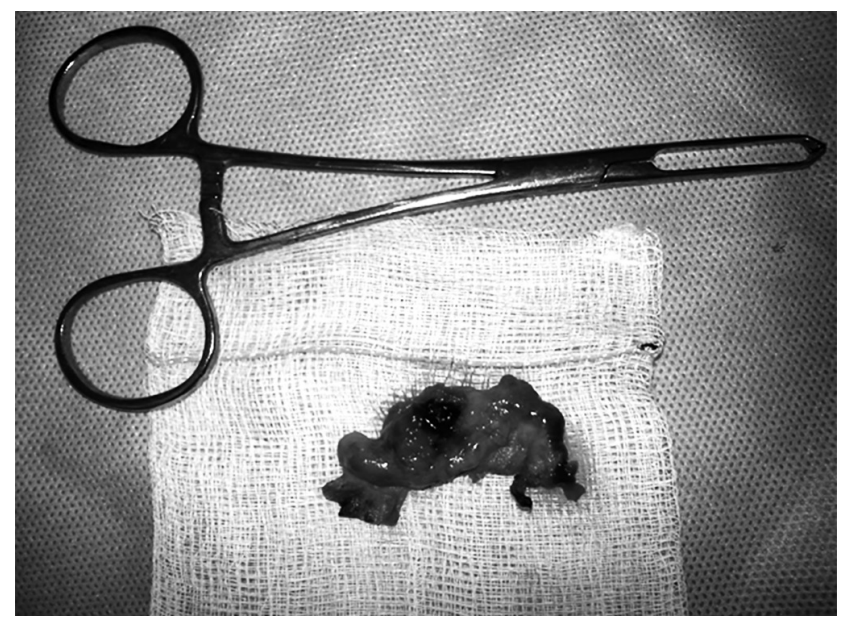

Fig. 4: Specimen showing right fallopian tube with products of conception

of a heterotopic pregnancy, it should be suspected in any patient who presents with lower abdominal pain in the early phase of an obvious intrauterine pregnancy following fertility treatment. ${ }^{8,9}$
Nowadays, the use of assisted reproductive technology and fertility agents, such as clomiphene citrate can increase a patient's risk of a heterotopic pregnancy probably due to the combined effects of hyperstimulation and the subsequent, simultaneous transfer of several embryos into the uterus with retrograde flow into the fallopian tubes. Indeed, any factor predisposing a patient to an increased risk of ectopic pregnancy and/or multiple gestations may contribute to heterotopic pregnancy. ${ }^{1-4,9}$ In one of our patients, pregnancy also occurred in association with ovulation induction.

Often, abdominal and pelvic ultrasonography fail to show the ectopic pregnancy or is misinterpreted because of the awareness of an already existing intrauterine pregnancy. ${ }^{3,4}$ But, demonstration of an intrauterine pregnancy is no longer a reliable indicator for excluding an ectopic pregnancy. ${ }^{4,7}$ If a patient has a beta subunit of human chorionic gonadotropin level of 1,500 mIU per $\mathrm{ml}$ or greater, but the transvaginal ultrasonography does not 
show an intrauterine gestational sac, ectopic pregnancy should be suspected.

Most ultrasonographic reports make no mention for a search of coexistent ectopic pregnancy, when evaluating intrauterine gestation, because a heterotopic pregnancy is still thought to be extremely rare and for this reason, almost all ectopic pregnancies are diagnosed by excluding an intrauterine pregnancy. ${ }^{1}$

Our cases also presented early in the pregnancy with a history of nausea, scant vaginal bleeding and lower abdominal pain. These symptoms are common in intrauterine pregnancy. There was also a delay in the detection of the ectopic pregnancy component, in the first case scenario, therefore diagnosis was not made until an ectopic pregnancy rupture had occurred and the patient developed hemoperitoneum and instability of vital signs. ${ }^{5}$ Although the primary ultrasonography helped to confirm the presence of an intrauterine pregnancy, it failed to identify the ectopic pregnancy, while a heterotopic pregnancy as a cause for abdominal pain should have been suspected immediately in our case.

The management of heterotopic pregnancy remains controversial. Surgical therapy has been the traditional mainstay but involves surgical and anesthetic risks to both the mother and intrauterine pregnancy. ${ }^{3}$ Studies suggest that laparoscopic management is preferred over laparotomy in patients with a suspected ectopic pregnancy, and with a documented intrauterine pregnancy because of minimal manipulation of the uterus., ${ }^{9,10}$

A nonsurgical approach can be used safely and effectively to manage patients who are clinically stable and where a heterotopic pregnancy is recognized relatively early in gestation. The successful nonsurgical management of six cases of heterotopic pregnancy using potassium chloride injection into the tubal ectopic pregnancy has been reported. ${ }^{3}$ In our case, if ectopic pregnancy had been diagnosed early, then it might have been possible to complete the surgery with the laparoscope, but because of hemodynamic instability in our case, an urgent laparotomy was arranged.

\section{CONCLUSION}

We can conclude that heterotopic pregnancy must always be suspected in patients who have a documented intrauterine pregnancy, who are presenting with abdomino pelvic pain in the first trimester, because just the presence of an intrauterine pregnancy can no longer be considered reassuring and a heterotopic pregnancy has to be ruled out. Thus, all patients with an intrauterine pregnancy should be given a comprehensive pelvic ultrasound to rule out the possibility of a simultaneous heterotopic pregnancy. Emphasis has to be given for prompt and immediate action, at the first sign which indicates a heterotopic pregnancy, such that we avoid missing this potentially life-threatening condition.

Treatment for patients with unruptured ectopic pregnancy may include expectant management, or medical management with methotrexate or surgery. Expectant management is appropriate only when beta subunit of human chorionic gonadotropin levels are low and declining. Initial levels determine the success of medical treatment. Surgical treatment is appropriate if ruptured ectopic pregnancy is suspected and if the patient is hemodynamically unstable.

Laparoscopic surgery has reached great heights at minimally invasive surgery, faster recovery and better results at the hands of an expert surgeon. Hence, laparoscopic management should definitely be considered in cases of hemodynamically stable patients instead of laparotomy.

\section{CONSENT}

Written informed consent was obtained from the patient for publication of this case report and accompanying images.

\section{REFERENCES}

1. Mishra A, Youssefzadeh D, Pare nte JT. Heterotopic pregnancy. Female Patient 1998;23:39-42.

2. Maalt ME, Dabbas MMN. Advanced heterotopic pregnancy. J Obstet Gynaecol 1999;19:677-678.

3. Scheiber MD, Cedars MI. Successful non-surgical management of a heterotopic an abdominal pregnancy following embryo transfer with cryopreserved-thawed embryos. Hum Reprod 1999;14:1375-1377.

4. Mistry BM, Balasubramaniam S, Silverman R, Sakabu SA, Troop BR. Heterotopic pregnancy presenting as an acute abdomen: a diagnostic masquerader. Am Surg 2000;66(3): 307-308.

5. Dessole S, Ruiu GA, Cherchi PL. Coexistence of a heterotopic pregnancy associated with a homolateral ovarian cyst in a patient submitted to elective abortion. Gynecol Obstet Invest 2000;49:277-278.

6. Dumesic DA, Damario MA, Session DR. Interstitial heterotopic pregnancy in a woman conceiving by in vitro fertilization after bilateral salpingectomy. Mayo Clin Proc 2001;76: 90-92.

7. Hill J. Assisted reproduction and the multiple pregnancy: increasing the risks for heterotopic pregnancy. J Diagn Med Sonogr 2003;19:258-260.

8. Archibong EI, Etuk SJ. Case report, heterotopic pregnancy following induction of ovulation. Trop J Obstet Gynecol 2002; 19:115-116.

9. Perkins JD, Mitchell MR. Heterotopic pregnancy in a large inner-city hospital: a report of two cases. J Natl Med Assoc 2004;96:363-366.

10. Siliva PD, Meisch Al. Laparoscopic treatment of heterotopic pregnancy. Am Assoc Gynecol Laparosc 1995;2:213-216. 